\title{
Incarcerated ovarian inguinal hernia in a 10-month-old girl
}

\author{
Jeffrey Lee MD, Nick Scampoli MD
}

Competing interests: None declared.

This article has been peer reviewed.

The authors have obtained patient consent.

Correspondence to: Jeffrey Lee, jeffrey.lee @ trilliumhealthpartners.ca

CMAJ 2015. DOI:10.1503 /cmaj.140823
$\Lambda$ healthy 10-month-old girl was brought to the emergency department by her parents because of the sudden appearance of a mass in her right groin. Her parents had noticed the mass while changing her diaper and commented that she became increasingly fussy when the area of swelling was examined.

The patient was neither lethargic nor vomiting. Two days earlier, she had sustained a lowgrade fever and had nonbloody diarrhea, which was thought to have been due to viral gastroenteritis, in addition to upper respiratory tract symptoms. The patient continued to void well and did not show signs of septicemia.

The patient's medical history was unremarkable, with no congenital anomalies or surgical procedures. She had been born by cesarean delivery with no complications, she took no medications and had no allergies, and all vaccinations were up to date.

The patient appeared well on physical examination and had normal vital signs (temperature $36.1^{\circ} \mathrm{C}$, pulse 145 beats/min, respiratory rate 28 breaths/min and oxygen saturation $98 \%$ on room air). Her skin was unremarkable, and her extremities were well perfused. The results of an examination of her head and neck were normal. The patient's lungs were clear, and the results of a cardiac examination were normal. Her abdomen was soft, nontender and showed no organomegaly. A soft, discrete mass $(1 \times 1 \mathrm{~cm})$ was palpable over the patient's right inguinal area. The patient became irritable upon palpation of the mass, and the mass was not reducible. We ordered ultrasonography to confirm a diagnosis of incarcerated inguinal hernia.

\section{KEY POINTS}

- An inguinal hernia in a female infant should prompt thorough investigation to determine whether there is any ovarian content.

- Ultrasonography is a safe and useful diagnostic tool.

- Irreducible ovaries are at high risk for subsequent torsion or strangulation; early surgical consultation and timely repair can reduce this risk.
Imaging confirmed a right indirect inguinal hernia containing the right ovary. Perfusion to the ovary was intact; however, we considered the risk of torsion to be high given the tenderness on examination. We obtained a pediatric surgical consult, and the patient was transferred for further evaluation and management.

The incarcerated hernia was successfully reduced, and the patient was discharged from hospital the same day. A follow-up appointment was made, and subsequent outpatient repair of the hernia was uneventful. The patient recovered well with no long-term sequelae.

\section{Discussion}

Inguinal hernias are common in infants and children. The reported frquency ranges between $1 \%$ and $13 \%$ and increases to about $30 \%$ in preterm infants. The incidence is highest during the first year of life, with more than one-third of cases occurring in infants aged less than six months. ${ }^{1-3}$ Inguinal hernias tend to affect male infants more often than female infants, with documented ratios ranging between $3: 1$ and 10:1.,

The incidence of an incarcerated ovary within an inguinal hernia is unclear in the literature. A recent review of a pediatric surgeon's practice from 1969 to 2004 involving 6361 infants and children with inguinal hernias found an overall incarceration rate of $9 \%$ among the 1018 female patients who underwent evaluation. ${ }^{5}$ Although this figure does not specifically refer to incarcerated ovaries, it has been reported that $82 \%$ of incarcerated inguinal hernias involve ovarian structures. ${ }^{6}$ In a review of 237 laparoscopically treated inguinal hernias in girls, ${ }^{7}$ Takehara and colleagues reported the presence of ovaries within incarcerated inguinal hernias to be $6 \%$. Thus, an accurate incidence of irreducible ovaries can only be estimated, and most likely ranges from $6 \%$ to $7 \%$ of inguinal hernias in girls. 


\section{Embryology}

Indirect inguinal hernias result from a variation of the inguinal canal during fetal development. At six weeks' gestation, germs cells begin to differentiate into testes or ovaries along the posterior abdominal wall. As the fetus elongates, the gonads migrate toward the internal inguinal ring. During the third month of gestation, the parietal peritoneum protrudes through the abdominal wall at the internal inguinal ring to form a diverticulum, known as the processus vaginalis.

In male fetuses, the testes begin to descend around seven months using the gubernaculum, a mesenchymal cord that attaches to the gonad to the mesonephros, as a guide through the inguinal canal. Once testicular descent is complete, the processus vaginalis obliterates. A similar process occurs in female fetuses, except that the descent remains intraperitoneal. The gubernaculum converts into the ovarian and round ligaments, and the corresponding processus vaginalis, called the Nuck diverticulum, eventually enters into the labia majora.

The processus vaginalis typically obliterates by eight months' gestation. However, persistence of the opening may subsequently result in hydroceles and inguinal hernias that present in infancy and childhood (Figure 1).,3

\section{Differential diagnosis}

Although inguinal masses are common in infants, the differential diagnosis is broad. Inguinal hernias account for most inguinal masses in both male and female pediatric patients, followed by lymphadenitis. In male patients, further consideration should be given to undescended

\footnotetext{
Box 1: Differential diagnosis for inguinal mass in infants 2,9

- Inguinal hernia (with or without involvement of the bowel, bladder, appendix, ovary, fallopian tube or uterus)

- Hydrocele (labia or spermatic cord)

- Lymphadenopathy

- Lymphadenitis

- Abscess

- Rhabodomyosarcoma

- Metastases

- Epidermal inclusion cyst

- Cystic lymphangioma

- Femoral hernia

- Ovarian torsion

- Undescended testes

- Testicular tumour

- Testicular torsion or testicular appendage
}

testes, hydrocele, testicular torsion and testicular tumour. In female patients, inguinal hernias containing ovarian content and labial hydrocele should be considered. Lymphadenitis, rhabdomyosarcoma and metastatic tumours should be included in the differential diagnosis for both sexes (Box 1). ${ }^{2,9}$

\section{Presentation}

Sliding inguinal hernias typically present as an intermittent bulge in the groin, scrotum or labia. It is especially evident during times of increased intra-abdominal pressure, such as crying or straining. With the patient positioned supine and undressed, the examiner can often find inguinal asymmetry or mass. When the bulge is not apparent, the examiner can employ various
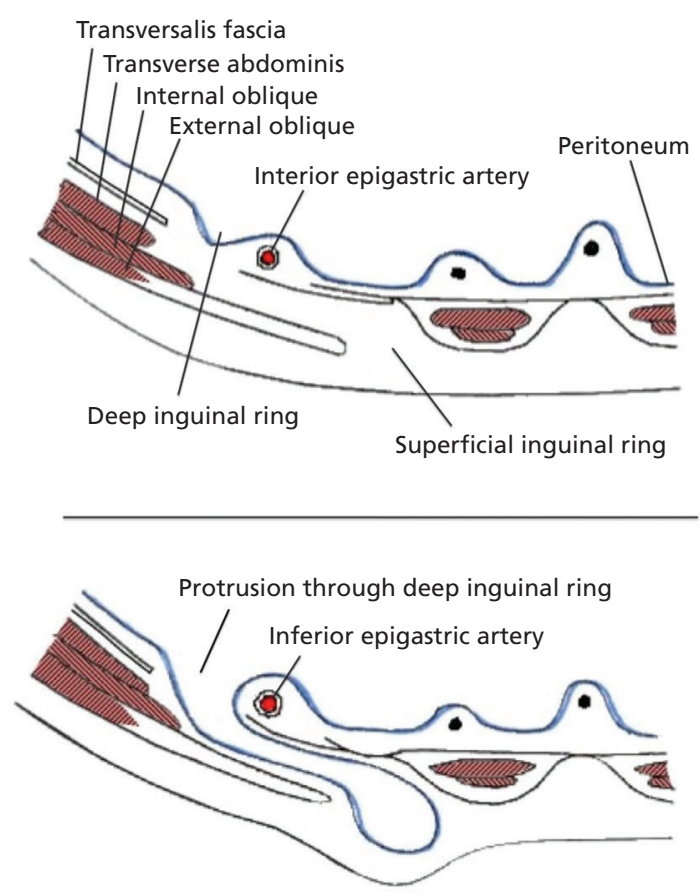

A. Indirect inguinal hernia

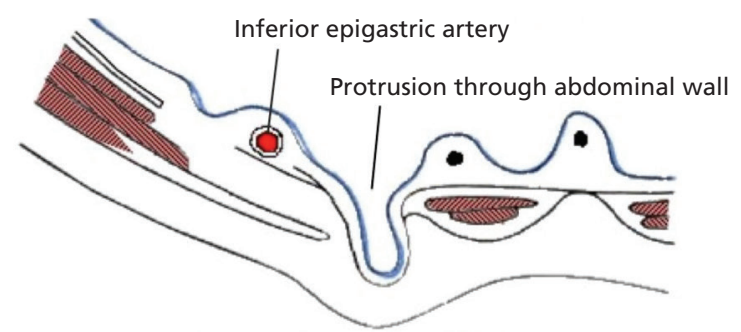

B. Direct inguinal hernia

Figure 1: Indirect inguinal hernias protrude through the deep inguinal ring lateral to the inferior epigastric artery (A). Direct inguinal hernias protrude through the abdominal wall medial to the inferior epigastric artery (B). Adapted, with permission, from O'Rahilly R, Müller F, Carpenter S, et al. Basic human anatomy: a regional study of human structure. Hanover (NH): Dartmouth Medical School; 2004. 
manoeuvres to try to increase intra-abdominal pressure. Infants can be allowed to strain or cry, and the older child can be positioned upright to perform a Valsalva manoeuvre or be instructed to cough or jump. This will often allow the mass to emerge through the external inguinal ring and present as a bulge. .,3 $^{2,}$

Incarcerated inguinal hernias occur when the contents cannot be reduced into the intraabdominal cavity. In this situation, the patient may present with a painful, erythematous and edematous mass. ${ }^{2}$ In addition, patients may have associated symptoms of irritability, decreased appetite, abdominal distention, vomiting and obstipation. ${ }^{3}$ If the hernia is not reduced, progressive worsening of symptoms will ensue as gangrene and tissue necrosis develop. ${ }^{6}$

\section{Management}

A critical step in managing inguinal hernias in female patients is to determine whether there is ovarian involvement. In very few cases, the uterus may be found in the sac. ${ }^{4}$ Ovarian tissue can usually be palpated as a small mobile mass, but it is often better identified with ultrasonography.

Management of the ovary within an inguinal hernia will be affected by its reducibility and clinical suspicion of strangulation or torsion. Unlike inguinal hernias involving the testes, strangulation by surrounding loops of intestine within the hernia sac is not thought to be the common mechanism of ovarian necrosis. Instead, the most noted primary pathological event is caused by torsion of the suspended ovary while in the hernia sac, similar to the "bell-clapper" anatomic variant that results in spontaneous testicular torsion, where there is insufficient fixation of the gonad. ${ }^{6}$

Early surgical intervention for the irreducible ovary is mainly guided by large retrospective chart reviews and case report recommendations, which document high rates of torsion or strangulation among patients with irreducible ovaries. The reported frequency of torsion in various case series ranges between $2 \%$ and $33 \% .^{5}$ In addition, no evidence exists to support a critical period that predicts when an ovary will undergo torsion after entrapment in a hernia. ${ }^{4}$

In cases where careful manual reduction is successful or incarceration is unlikely, surgical repair can usually be done on an outpatient basis a few weeks after presentation. If reduction proves to be difficult or requires sedation, early surgical repair may be required. ${ }^{3}$

Situations in which surgery may need to be delayed include low birth weight, premature birth or the presence of congenital heart disease, pulmonary disease, sepsis or metabolic disease, because these conditions increase the risks associated with undergoing anesthesia. ${ }^{3}$

Irreducible ovarian content in an inguinal hernia should prompt the clinician to consider the risk of torsion or strangulation. Pain elicited over the mass may represent early signs of decreased perfusion, and suspicion for a twisted ovary should be high. Prompt referral to a pediatric surgeon for timely surgical repair is recommended to reduce the chance of torsion and potentially save viable reproductive tissue.

\section{References}

1. Ravi K, Hamer DB. Surgical treatment of inguinal herniae in children. Hernia 2003;7:137-40.

2. Kapur P, Caty MG, Glick PL. Pediatric hernias and hydroceles. Pediatr Clin North Am 1998;45:773-89.

3. Palmer LS. Hernias and hydroceles. Pediatr Rev 2013;34:457-64.

4. Okada T, Sasaki S, Honda S, et al. Irreducible indirect inguinal hernia containing uterus, ovaries, and fallopian tubes. Hernia 2012;16:471-3.

5. Ein SH, Njere I, Ein A. Six thousand three hundred sixty-one pediatric inguinal hernias: a 35-year review. J Pediatr Surg 2006; 41:980-6.

6. Merriman TE, Auldist AW. Ovarian torsion in inguinal hernias. Pediatr Surg Int 2000;16:383-5.

7. Takehara H, Hanaoka J, Arakawa Y. Laparoscopic strategy for inguinal ovarian hernias in children: when to operate for irreducible ovary. J Laparoendosc Adv Surg Tech A 2009;19 (Suppl 1):S129-S131.

8. O'Rahilly R, Müller F, Carpenter S, et al. Basic human anatomy: a regional study of human structure. Hanover (NH): Dartmouth Medical School; 2004. Available: www.dartmouth.edu/ humananatomy/ (accessed 2014 Oct. 14).

9. Hennelly K, Shannon M. A 3-month-old female with an inguinal mass. J Emerg Med 2011;40:33-6.

Affiliations: Department of Family Medicine (Lee), Trillium Health Centre, Mississauga, Ont., and the Department of Emergency Medicine (Scampolli), Credit Valley Hospital, Mississauga, Ont.

Contributors: Both authors contributed to the writing and revision of the manuscript and approved the final version submitted for publication.

Acknowledgements: The authors thank the patient's family for providing consent to report on their child's case.

The section Cases presents brief case reports that convey clear, practical lessons. Preference is given to common presentations of important rare conditions, and important unusual presentations of common problems. Articles start with a case presentation (500 words maximum), and a discussion of the underlying condition follows (1000 words maximum). Visual elements (e.g., tables of the differential diagnosis, clinical features or diagnostic approach) are encouraged. Written consent from patients for publication of their story is a necessity and should accompany submissions. See information for authors at www.cmaj.ca. 